\title{
Electrically driven, phosphor-free, white light-emitting diodes using gallium nitride-based double concentric truncated pyramid structures
}

\author{
Seung-Hyuk Lim ${ }^{1}$, Young-Ho Ko ${ }^{1,2}$, Christophe Rodriguez ${ }^{1,3}$, Su-Hyun Gong ${ }^{1}$ and Yong-Hoon Cho ${ }^{1}$ \\ White light-emitting diodes (LEDs) are becoming an alternative general light source, with huge energy savings compared to \\ conventional lighting. However, white LEDs using phosphor(s) suffer from unavoidable Stokes energy converting losses, higher \\ manufacturing cost, and reduced thermal stability. Here, we demonstrate electrically driven, phosphor-free, white LEDs based on \\ three-dimensional gallium nitride structures with double concentric truncated hexagonal pyramids. The electroluminescence spectra \\ are stable with varying current. The origin of the emission wavelength is studied by cathodoluminescence and high-angle annular dark \\ field scanning transmission electron microscopy experiments. Spatial variation of the carrier injection efficiency is also investigated by \\ a comparative analysis between spatially resolved photoluminescence and electroluminescence.
}

Light: Science \& Applications (2016) 5, e16030; doi:10.1038/Isa.2016.30; published online 12 February 2016

Keywords: indium gallium nitride; light-emitting diodes; phosphor-free; three-dimensional structures; white color

\section{INTRODUCTION}

Group III-nitride semiconductor materials have been widely used in various optoelectronic devices, including light-emitting diodes (LEDs), laser diodes, and photodetectors. With technological advances in group III-nitride semiconductors ${ }^{1-3}$, white LEDs using phosphor(s) that replace existing lighting sources have already been commercialized. However, phosphor-free white light sources are still in demand due to the drawbacks of using phosphor, such as Stokes energy conversion loss, high manufacturing cost, and reduced thermal stability of the phosphor-based LEDs ${ }^{3,4}$. Therefore, to achieve phosphor-free broadband LEDs, two-dimensional (2D) film $^{5-7}$ and three-dimensional (3D) gallium nitride $(\mathrm{GaN})$ structures ${ }^{8-12}$ have been studied using metal-organic vapor-phase epitaxy (MOVPE).

3D GaN structures offer several advantages, such as (i) improving the quality of multi-quantum wells (MQWs) due to the use of a selective-area epitaxial growth (SAG) technique ${ }^{13}$; (ii) reducing the quantum-confined Stark effect (QCSE) by using semi- and/or nonpolar facets ${ }^{14,15}$; (iii) varying the indium composition, for the broad spectrum, as the distance changes from the mask because of different diffusion lengths between indium and gallium adatoms ${ }^{16,17}$; and (iv) enhanced light extraction efficiency because 3D GaN structures have textured surfaces ${ }^{18}$. Although some groups have demonstrated phosphor-free broad spectrum LEDs using 3D GaN structures grown by MOVPE, the realization of phosphor-free white LEDs has proved to be more challenging. In the previously reported results regarding phos- phor-free white LEDs, a dramatic color coordinate shift was observed with changing injection current ${ }^{8,9,19,20}$. For the purpose of a white light source, it is important to determine the origin of the color emission change and to achieve stable color emission.

Here, we report phosphor-free white LEDs that consistently emit white light with varying injection current. We intentionally designed the pattern and size to achieve double concentric, truncated pyramid fabricated (DCTP) structures with a truncated hexagonal pyramid surrounded by a hexagonal ring. We also investigated the origin of broadband emission and discuss the carrier injection efficiency for both spatially resolved micro-electroluminescence $(\mu-\mathrm{EL})$ and micro-photoluminescence ( $\mu$-PL).

\section{MATERIALS AND METHODS}

\section{Growth and fabrication of DCTP LEDs}

The $n$-GaN template was grown on a c-plane sapphire substrate by MOVPE. A $30 \mathrm{~nm} \mathrm{Si}_{3} \mathrm{~N}_{4}$ mask layer (Figure 1a) was fabricated on an $n$-GaN template by plasma-enhanced chemical vapor deposition and a UV-lithography technique. The $\mathrm{Si}_{3} \mathrm{~N}_{4}$ mask layer patterned with openings of concentric circles consisting of a central hole and a ring is schematically illustrated in Figure 1a. The hole diameter $\left(d_{\text {hole }}\right)$, inner ring diameter $\left(d_{\mathrm{in \_ ring}}\right)$, outer ring diameter $\left(d_{\text {out_ring }}\right)$, and center-to-center distance $\left(d_{\text {pitch }}\right)$ were $3,9,15$, and $18 \mu \mathrm{m}$, respectively. After SAG of an $n$-GaN layer at $1040{ }^{\circ} \mathrm{C}$ on the above-patterned $\mathrm{Si}_{3} \mathrm{~N}_{4}$ mask, we achieved DCTP structures. Figure $1 \mathrm{~b}$ schematically illustrates a cross-sectional view of the DCTP-structured LEDs. The height

\footnotetext{
${ }^{1}$ Department of Physics and KI for the NanoCentury, Korea Advanced Institute of Science and Technology, 291 Daehak-ro, Yuseong-gu, Daejeon 34141, Korea;

2Present address: Electronics and Telecommunications Research Institute (ETRI), 218 Gajeong-ro, Yuseong-gu, Daejeon 34129, Korea;

${ }^{3}$ Present address: The Laboratoire Nanotechnologies Nanosystèmes (LN2) - CNRS UMI-3463, Institut Interdisciplinaire d'Innovation Technologique (3IT), Université de Sherbrooke, 3000 boulevard Université, J1K OA5, QC, Canada

Correspondence: YH Cho, Email: yhc@kaist.ac.kr

Received 14 May 2015; revised 18 September 2015; accepted 25 September 2015; accepted article preview online 26 September 2015
} 


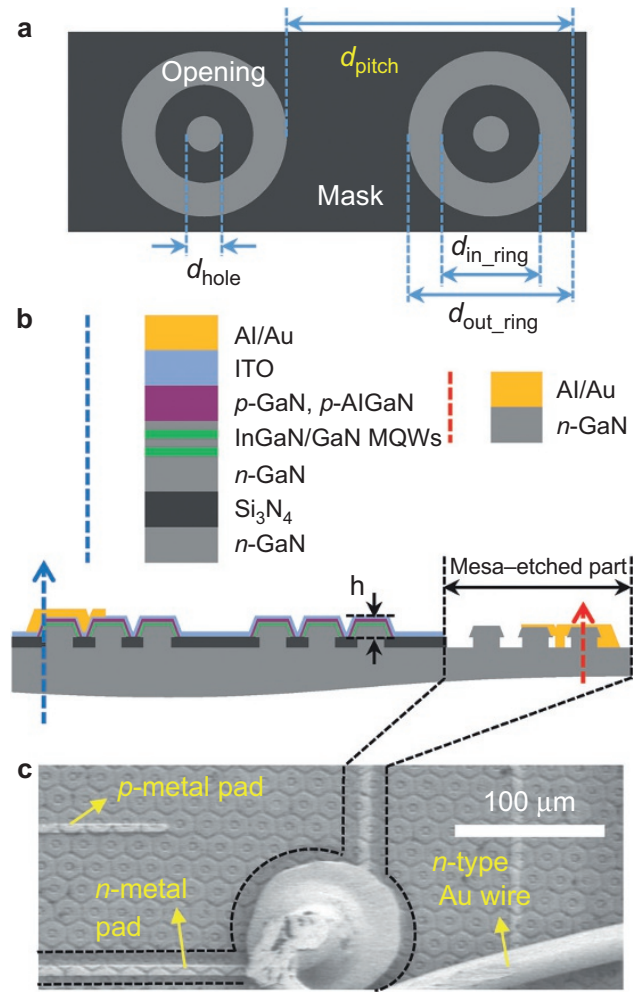

Figure 1 Device overview: (a) Top view mask schematic for SAG of DCTP LEDs. Dark gray and light gray areas are the $\mathrm{Si}_{3} \mathrm{~N}_{4}$ mask and the opening part, respectively. (b) Cross-sectional view schematic and layer contents for both the LED and mesa-etched area (drawing not to scale). (c) Top view SEM image of the DCTP LED after the metal contact process.

of the $n$-GaN DCTP structure is $1.3 \mu \mathrm{m}$, which indicates that this structure has a lower aspect ratio compared with other types of $3 \mathrm{D}$ LEDs, such as rods, pyramids, and annular structures. Five pairs of InGaN/GaN MQWs at $680^{\circ} \mathrm{C} / 850^{\circ} \mathrm{C}, p-\mathrm{AlGaN}$, and $p-\mathrm{GaN}$ layers were heteroepitaxially grown on $n$-GaN DCTP structures. The V/III ratios of $n$-GaN DCTP, InGaN quantum wells, and GaN quantum barriers were 60,4000 , and 1000, respectively. After mesa etching (LED chip size: $1.2 \mathrm{~mm} \times 0.6 \mathrm{~mm}$ ), a $55-\mathrm{nm}$ thick (based on c-plane) indium tin oxide (ITO) layer was deposited on the DCTP LEDs. An Al/Au layer was used for $n$ - and $p$-type electrodes. Figure $1 c$ shows a top view scanning electron microscope (SEM) image of a DCTP LED after the $\mathrm{Au}$ wire bonding process, and black dotted lines indicate the mesa-etched part. To calculate the injected current density, the 3D geometrical area of $p$-GaN was deduced by top view SEM image instead of the conventionally used 2D mesa-etched area. By considering the overall grown area on the mask and approximately $60^{\circ}$ inclined semi-polar facets $\left(\{10 \overline{1} 1\}: 61.9^{\circ},\{11 \overline{2} 2\}: 58.4^{\circ}\right)^{21}$, the $p$-GaN area (i.e., $0.754 \mathrm{~mm}^{2}$ ) of the 3D DCTP structure is approximately 1.05 times (see the Supplementary Information) larger than the 2D mesa area (i.e., $0.720 \mathrm{~mm}^{2}$ ).

\section{Cathodoluminescence and structural characterization}

Spatial optical characterization on DCTP LED was performed at room temperature by cathodoluminescence (CL) (monoCL4, Gatan, Inc., Pleasanton, CA, USA) equipment connected to an SEM, where the acceleration voltage was $10 \mathrm{kV}$. A transmission electron microscopy specimen was prepared using the focused ion beam technique. The DCTP structure was also observed by spherical aberration-corrected,

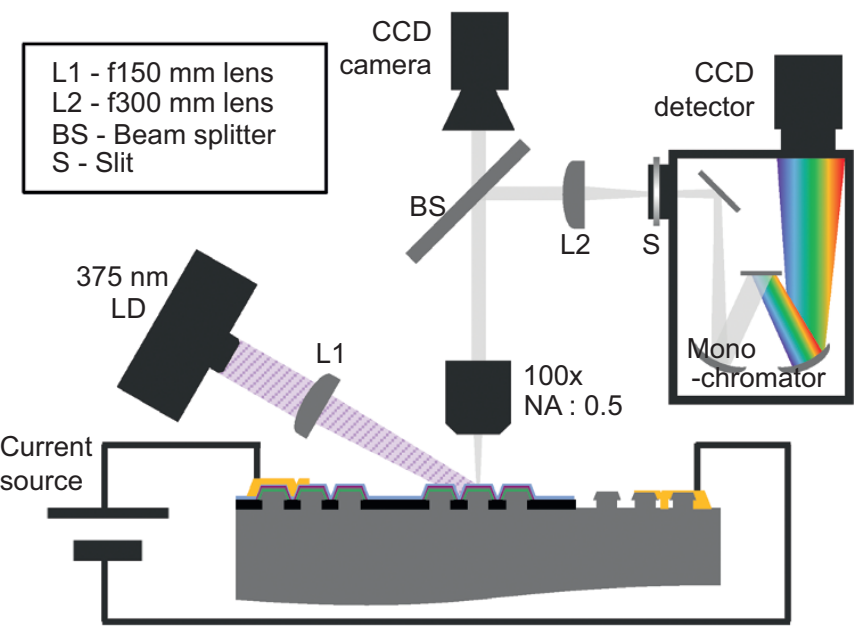

Figure 2 Setup schematic of $\mu$-PL and $\mu$-EL experiments. The DCTP structure was globally excited and locally detected for both $\mu$-EL and $\mu-P L$. The optics for the detection setup are shared, and both experiments were conducted at room temperature.

high-angle annular dark-field scanning transmission electron microscopy (HAADF-STEM) (JEM-ARM200F, JEOL, Ltd., Tokyo, Japan). To estimate the indium composition, the image contrast in the HAADF-STEM image scales and the atomic number, $Z$, were used ${ }^{22}$. Secondary ion mass spectrometry, which is frequently used to analyze the chemical composition in conventional $2 \mathrm{D}$ film, is not able to extract the indium molar fraction of the InGaN layers in 3D structure LEDs. The values from energy-dispersive X-ray spectrometry are underestimated because the information of the InGaN layer is detected together with that of the surrounding GaN barrier. It is therefore likely that an analytical method for checking the indium composition deduced from the HAADF-STEM intensity has higher spatial resolution than the other measurement techniques.

\section{$\mu$-PL and $\mu$-EL characterization}

To investigate the spatial variation of the electron-hole injection efficiency in a single DCTP structure, $\mu$-EL was conducted, and the results were compared with those from $\mu$-PL at room temperature. Figure 2 schematically illustrates the experiment setup for $\mu$-EL and $\mu$-PL. The DCTP structure was globally excited and locally detected for both $\mu$-EL and $\mu$-PL. Resonant PL was also conducted to excite the InGaN layer only. A microscope objective lens with a long working distance $(100 \times, \mathrm{NA}=0.5$, Mitutoyo Corporation, Kawasaki, Japan) was used to spatially collect (approximately $1 \mu \mathrm{m}^{2}$ ) the PL and EL emission from the DCTP structure in the normal direction. For global resonant excitation, a $375 \mathrm{~nm}$ semiconductor laser diode focused with a $150 \mathrm{~mm}$ macro lens and a source meter (Keithley 2400, Tektronix, Inc., Beaverton, OR, USA) were used for optical and electrical excitation, respectively. $\mu$-PL and $\mu$-EL spectra were measured using a monochromator (Acton SP2500, Princeton Instruments Corporation, Trenton, NJ, USA) in conjunction with a $2 \mathrm{D}$, charge-coupled device detector with a grating of 300 lines $\mathrm{mm}^{-1}$.

\section{RESULTS AND DISCUSSION}

\section{DCTP structure geometries}

DCTP structures have multifacets, including $\{10 \overline{1} 1\},\{11 \overline{2} 2\},(0001)$, and $\{11 \overline{2} 0\}$ facets, as shown in the top view SEM image presented in Figure 3 (and Figure $4 \mathrm{a}$ ). As reported by Leung et al. ${ }^{23}$, because the 


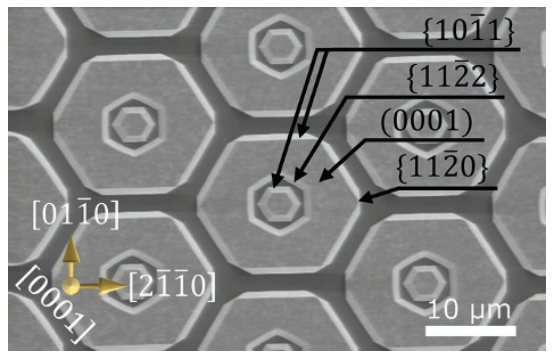

Figure 3 Top view SEM image of the DCTP LED after SAG. A DCTP structure has $\{11 \overline{2} 0\},(0001),\{11 \overline{2} 2\}$, and $\{10 \overline{1} 1\}$ facets.

concave growth front will be dominated by the fast-growing facet (i.e., the $\{11 \overline{2} 2\}$ facet), the diameter of the inner hexagonal ring (i.e., the concave growth front) decreases to $5 \mu \mathrm{m}$, whereas the diameters of the inner truncated hexagonal pyramid and the outer hexagonal ring (i.e., the convex growth front) slightly increase during the growth of $n$-GaN. To fabricate phosphor-free white LEDs, several facets and harmonious proportions of these facets are required. Furthermore, it is important to make 3D structures with lower height for easier and stable fabrication; otherwise, a planarization step may be required to deposit a conducting and/or metal layer smoothly. We emphasize that the patterns and the sizes of the DCTP structures have been intentionally designed to achieve white color emission from a single $3 \mathrm{D}$ structure that has a lower height.

\section{Emission characteristics of growth facets and their origin}

Figure 4a shows the top view SEM image of a single DCTP structure where the yellow points indicate the excitation position for CL spectra plotted in Figure 4b. The GaN band-edge emission at $366 \mathrm{~nm}$ and a broadband emission at approximately $560 \mathrm{~nm}$ were observed for all CL spectra. The CL peak wavelength from the MQWs on the $\{10 \overline{1} 1\}$ and
$\{11 \overline{2} 2\}$ semi-polar facets was approximately $400 \mathrm{~nm}$, whereas the emission from MQWs on the (0001) polar facet had a much longer wavelength of $560 \mathrm{~nm}$. Furthermore, we found an additional CL emission with a peak wavelength of $470 \mathrm{~nm}$ from MQWs on the $\{11 \overline{2} 0\}$ facet. To clarify the origin of these emission peaks, monochromatic CL mapping images were taken at wavelengths of 410,470 , and $560 \mathrm{~nm}$, as shown in Figure $4 \mathrm{c}-4 \mathrm{e}$, respectively. The emission of MQWs on the $\{11 \overline{2} 0\}$ facet is clearly distinguished from MQWs on other facets (Figure 4d).

A bird's eye view SEM image is shown in Figure 5a. For further study, a transmission electron microscopy specimen, indicated by the red line in Figure 5a, was prepared. Figure 5b shows a HAADF-STEM image of a cross-sectional view of the specimen. InGaN/GaN MQWs and $p$-AlGaN layers were clearly formed for both the (0001) and $\{11 \overline{2} 0\}$ facets. High magnification HAADF-STEM images of InGaN/ GaN MQWs and $p$-AlGaN on (0001), $\{11 \overline{2} 0\}$, and the joints between them are shown in Figure $5 c, 5 e$, and $5 d$, respectively. The thicknesses of the InGaN layers on (0001) and $\{11 \overline{2} 0\}$ are approximately $3.5 \mathrm{~nm}$ and $3 \mathrm{~nm}$, respectively. The HAADF-STEM intensity of MQWs on (0001) and $\{11 \overline{2} 0\}$ facets was taken along the yellow and green arrow directions from Figure $5 \mathrm{c}$ and $5 \mathrm{e}$ and plotted in Figure $5 \mathrm{f}$ and $5 \mathrm{~g}$, respectively. The cyan minor grid of Figure $5 \mathrm{f}$ and $5 \mathrm{~g}$ is of the same increment. The indium content inside the InGaN layers on the (0001) and $\{11 \overline{2} 0\}$ facets was extracted by Equation (1):

$$
I_{\mathrm{InGaN}} / I_{\mathrm{GaN}}=\frac{x Z_{\mathrm{In}}^{\varepsilon}+(1-x) Z_{\mathrm{Ga}}^{\varepsilon}+Z_{N}^{\varepsilon}}{Z_{\mathrm{Ga}}^{\varepsilon}+Z_{N}^{\varepsilon}}
$$

where $I$ is the HAADF-STEM intensity, $x$ is the elemental concentration in atomic percent, $Z$ is the atomic number, and $\varepsilon$ is a factor depending on the collection angle of the HAADF-STEM detector. The exponent was calibrated via energy dispersive spectroscopy ${ }^{24}$, and the average value of the indium composition $x$ was $0.232 \pm$ 0.052 for InGaN layers on the $(0001)$ facet and $0.120 \pm 0.009$ for
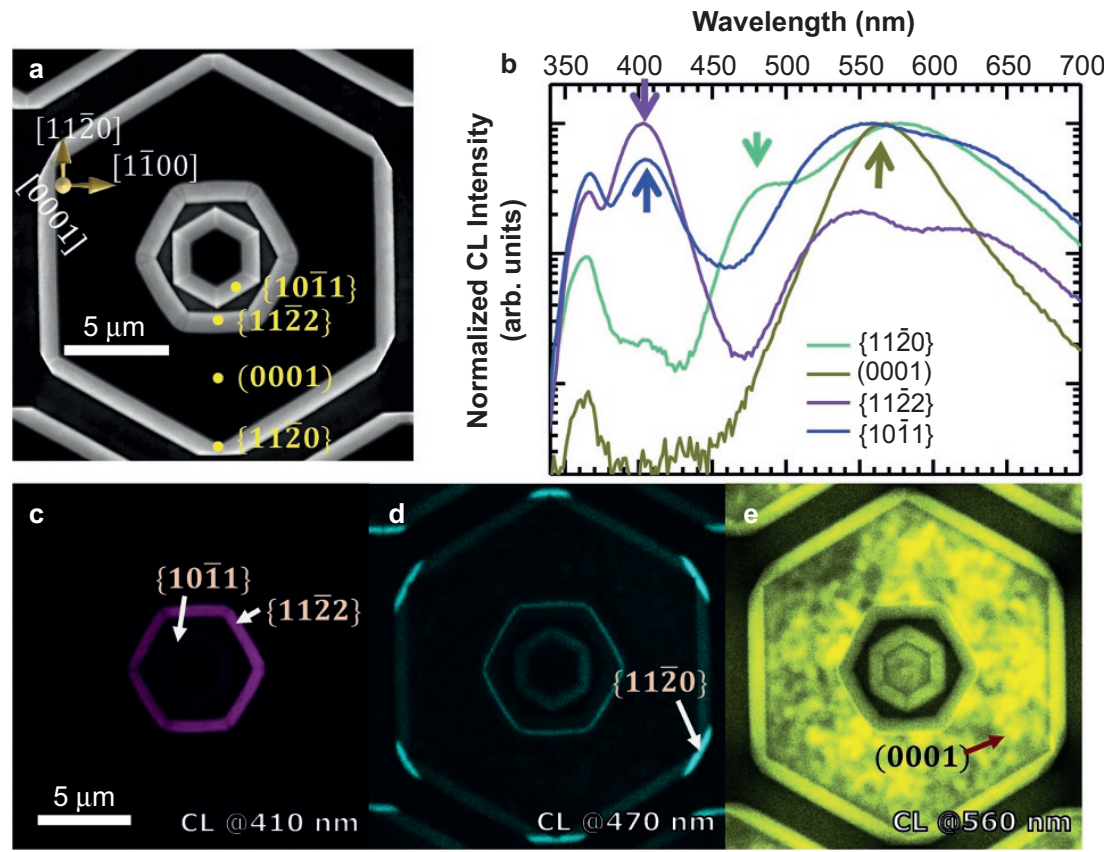

Figure 4 SEM image, spatially resolved CL spectra, and monochromatic CL images of the DCTP structure. (a) Top view SEM image of the DCTP structure. The yellow points indicate the excitation positions of the CL spectra. (b) The normalized CL spectra of InGaN/GaN MQWs on the $\{11 \overline{2} 0\},(0001),\{11 \overline{2} 2\}$, and $\{10 \overline{1} 1\}$ facets. The arrows indicate the specific emissions for each facet. Monochromatic CL mapping images taken at wavelengths of (c) $410 \mathrm{~nm}$, (d) $470 \mathrm{~nm}$, and (e) $560 \mathrm{~nm}$. 

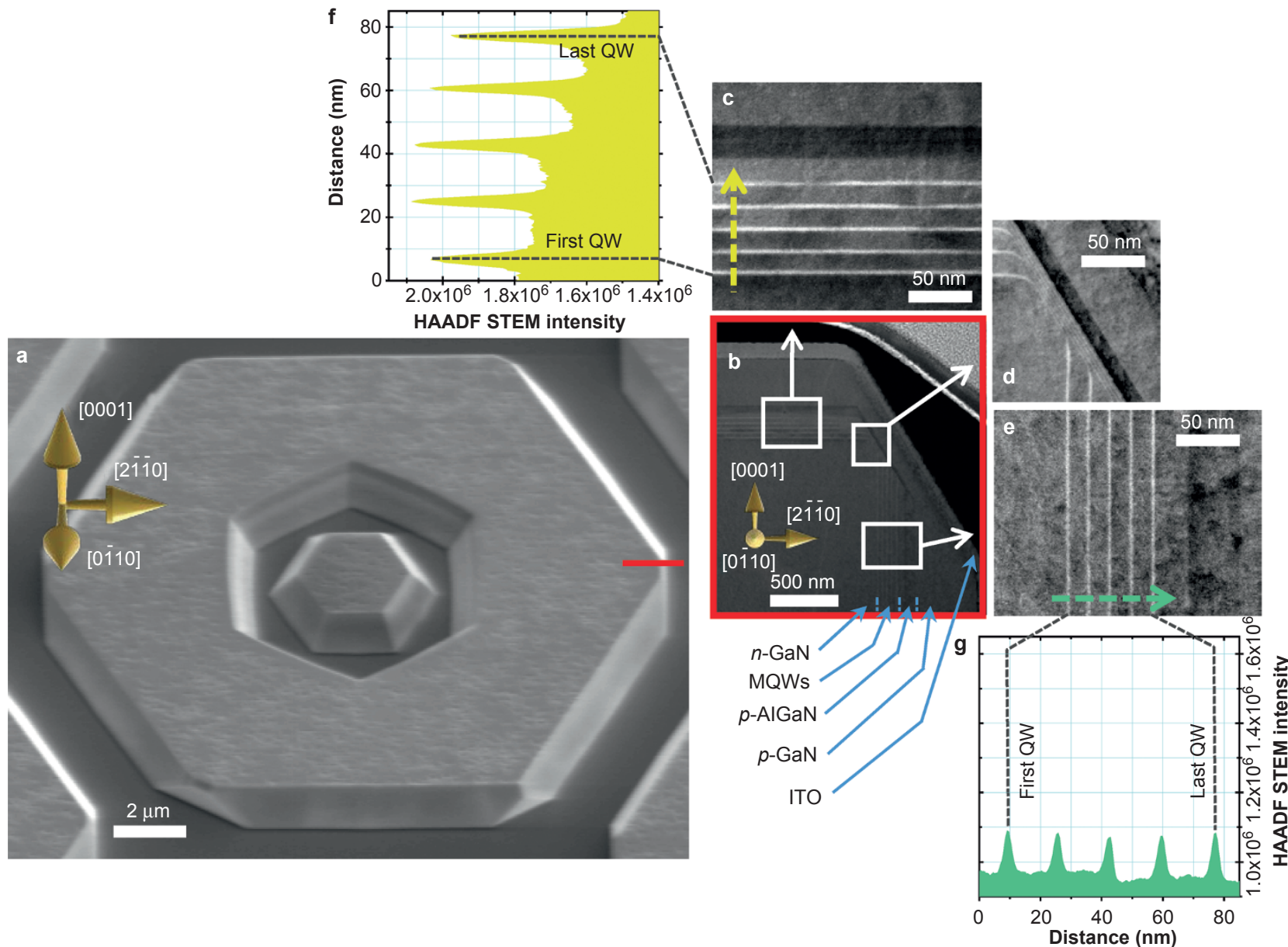

Figure 5 Structural characterization and indium composition analysis. (a) Bird's eye view SEM image of the DCTP structure. The red line indicates the position where the HAADF-STEM sample is inspected. HAADF-STEM images of (b) overview, (c) MQWs on the (0001) facet, (d) joint area, and (e) MQWs on the $\{11 \overline{2} 0\}$ facet. Yellow and green arrows indicate the scan direction and its range for the HAADF-STEM intensity of (f) MQWs on the (0001) facet and (g) MQWs on the $\{11 \overline{20}$.

InGaN layers on the $\{11 \overline{2} 0\}$ facet. InGaN MQWs on the (0001) facet have higher indium composition, greater well thickness, and a higher QCSE than those on $\{11 \overline{2} 0\}$. All factors push the QW emission to longer wavelength for InGaN QWs on the (0001) facet.

\section{Spatially resolved characterization method for carrier injection path}

Panchromatic (PAN) $\mu$-PL and $\mu$-EL images are shown in Figure $6 a$ and $6 \mathrm{c}$, and a $2 \mathrm{D}$ image of the line scan spectrum is shown in Figure $6 \mathrm{~b}$ and $6 \mathrm{~d}$, respectively. All images in Figure $6 \mathrm{a}-6 \mathrm{~d}$ are normalized. Although the excitation power of the $\mu$-PL (the injection current of $\mu$-EL) experiments was adjusted from $10 \mathrm{~mW}$ to $30 \mathrm{~mW}(10 \mathrm{~mA}-100$ $\mathrm{mA}$ with an LED chip size of $0.72 \mathrm{~mm}^{2}$ ), spatially resolved optical images and spectra were not significantly changed. Before obtaining the line-scan spectrum images, a slit was inserted in front of the monochromator to select the image area (between the black vertical lines in Figure $6 \mathrm{a}$ and $6 \mathrm{c}$ ), which is parallel to [1120], whereas PAN images were taken without the slit.

First, PAN images under $\mu$-PL and $\mu$-EL (Figure $6 \mathrm{a}$ and $6 \mathrm{c}$ ) conditions were compared. The PAN image under the $\mu$-EL condition (Figure 6c) shows much higher intensity emission at the boundary between the (0001) and $\{10 \overline{1} 1\}$ facets; however, the image under the $\mu$-PL condition (Figure $6 a$ ) shows a relatively homogeneous emission over the entire DCTP area. Because the sample and the detection setup were identical, similar internal quantum efficiency, light extraction efficiency, and emission collecting and detecting efficiency should be taken into account after the exciton generation, except for the carrier injection efficiency. We therefore found that the carrier injection efficiency is spatially different, whereas the photon injection is relatively uniform. The large spatial variation of the carrier injection efficiency is strongly related to the different thicknesses of the $p$-GaN layer, as shown in Figure 5b. Because of the growth rate change depending on the different facets, the $p$-GaN resistance between ITO and MQWs would vary locally. Consequently, higher hole injection efficiency occurs at a thinner $p$-GaN layer (i.e., an edge between a (0001) facet and a semi-polar or $\{11 \overline{2} 0\}$ facet). Figure $6 \mathrm{~b}$ and $6 \mathrm{~d}$ shows the line-scan PL and EL spectra along the vertical positions. The emissions from 400 to $450 \mathrm{~nm}, 450$ to $520 \mathrm{~nm}$, and 520 to $640 \mathrm{~nm}$ indicate MQWs on semi-polar, $\{11 \overline{2} 0\}$, and (0001) facets, respectively. Unlike the $\mu$-PL results (Figure $6 \mathrm{~b}$ ), the $\mu$-EL (Figure $6 \mathrm{~d}$ ) spectral image has a brighter position at the boundary between the semi-polar or $\{11 \overline{2} 0\}$ facets and the (0001) facet, as illustrated by the white dashed line in Figure 6d. The difference in the emission region from the (0001) facet (i.e., $520 \mathrm{~nm}-640 \mathrm{~nm}$ ) between the $\mu$-PL and $\mu$-EL images is evidence that the hole injection efficiency depends on the $p$-GaN thickness over the whole area. 


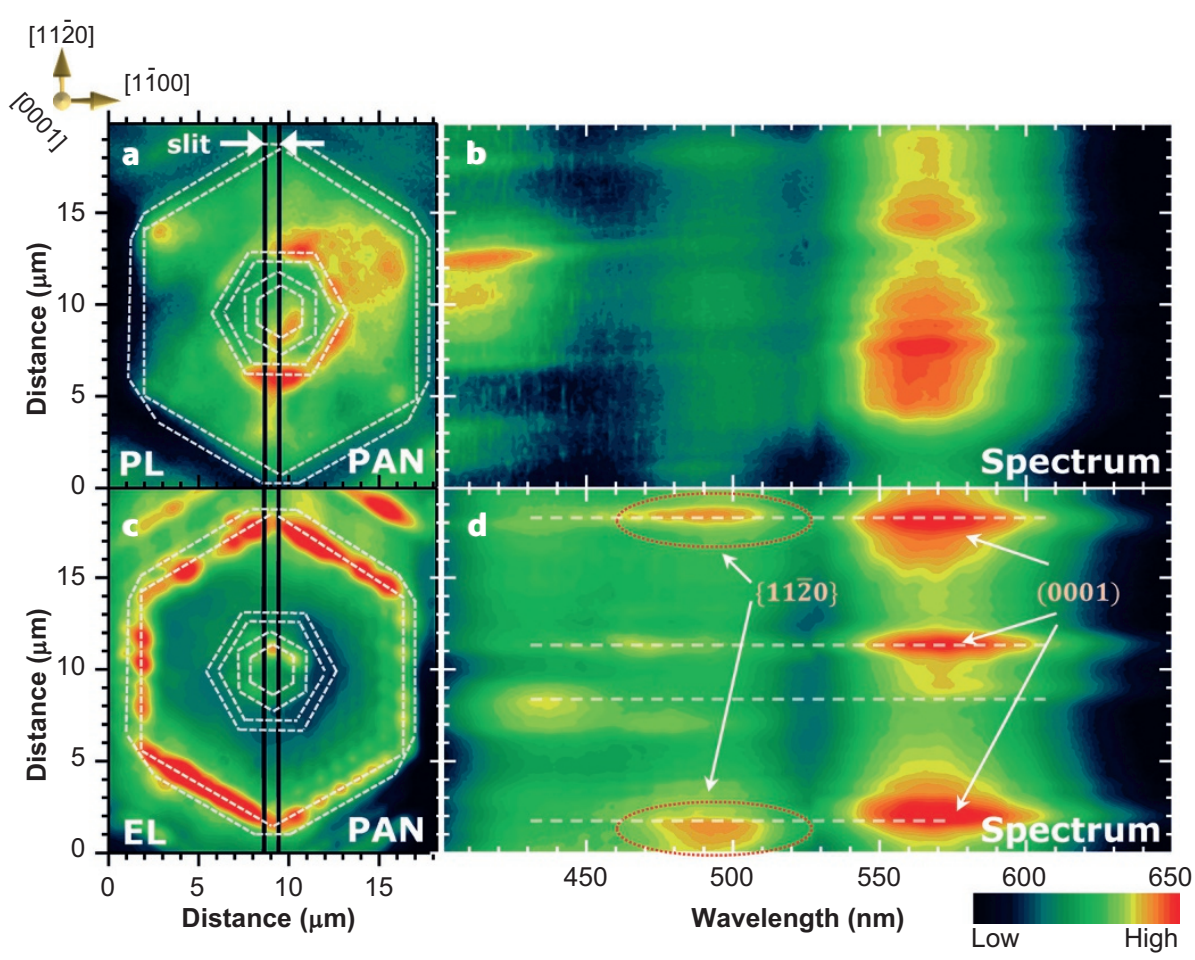

Figure 6 Optical characterization of the DCTP LEDs. (a) $\mu$-PL PAN image and (b) line selected $\mu$-PL spectrum image of the DCTP LEDs. (c) $\mu$-EL PAN image and (d) line selected $\mu$-EL spectrum image of the DCTP LEDs.

\section{Ensemble luminescence characteristic of DCTP LEDs}

From temperature-dependent PL data, a luminescence efficiency of approximately $3 \%$ was estimated for the entire spectrum $(380 \mathrm{~nm}-700$ $\mathrm{nm}$ ) emitting from MQWs formed on various facets of DCTP LEDs (not shown here). Ensemble EL spectra were obtained under varying current from $10 \mathrm{~mA}$ to $100 \mathrm{~mA}$ for DCTP LEDs, as shown in Figure 7, and the Commission Internationale de l'Eclairage (CIE)-1931 coordinates of the corresponding current are plotted in the inset of Figure 7 and are presented in Table 1. Because the emission of MQWs on the $\{11 \overline{2} 0\}$ facet is clearly distinguished from MQWs on other facets, the single DCTP structure is not only able to emit white light but also reaches a high color rendering index $(\mathrm{CRI}) \mathrm{Ra}(\sim 75 \%)$, which was calculated from ensemble EL spectra (Figure 7). The CIE coordinates do not show significant changes with increasing current because at the low-current density region, due to the lesser thickness of the $p$-GaN layer, hole injection starts at the joint area (i.e., an edge between the (0001) facet and the semi-polar or $\{11 \overline{2} 0\}$ facet), as shown in Figure $5 \mathrm{~d}$. The area of hole injection then isotopically increases to the direction of each facet (i.e., semi-polar, $\{11 \overline{2} 0\}$, and (0001)) with increasing current. Because the portion of injected holes on each facet is not varied, DCTP LEDs were able to emit stable white light. A photographic image was taken and is displayed in the inset of Figure 7. The white emission properties (e.g., CIE coordinates and CRI value) can be adjusted by changing: (i) the size and design of the mask patterning; (ii) the growth time and V/III ratio of the $n$-GaN DCTP; and (iii) the growth conditions of the active layers (e.g., growth temperature, flow of group-III precursors).

\section{CONCLUSIONS}

We demonstrated electrically driven, phosphor-free white LEDs with DCTP structures grown by MOVPE. The patterns and sizes of the

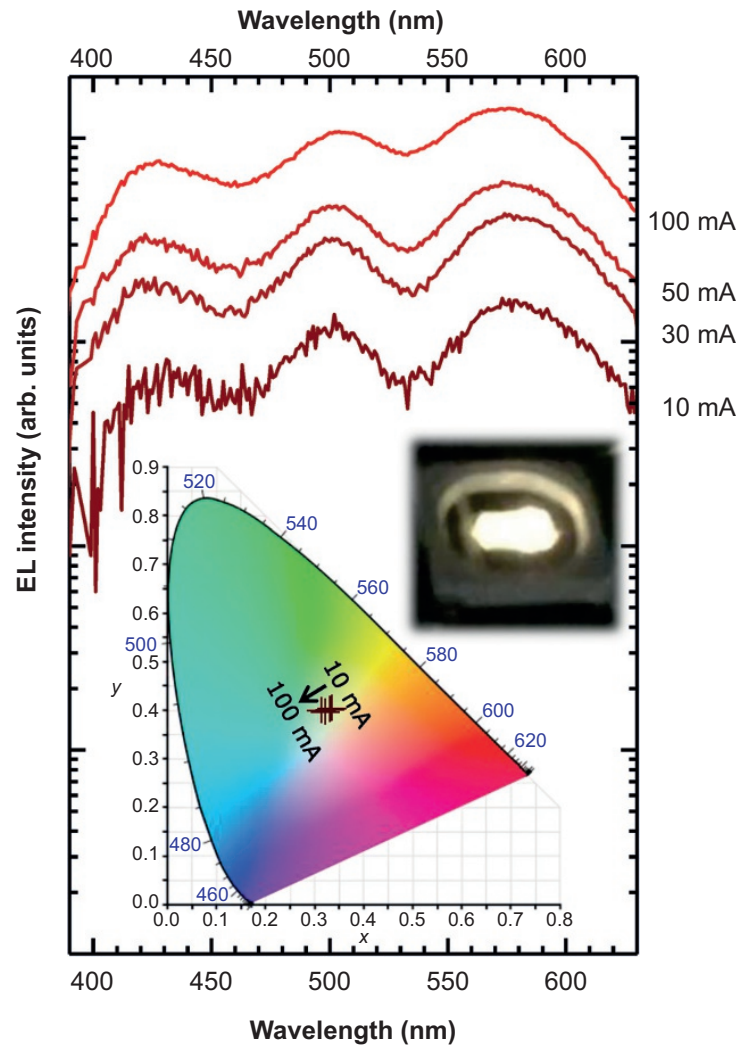

Figure 7 Current dependent ensemble EL spectra of the DCTP LEDs. The inset shows a photographic image and color coordinates for currents between $10 \mathrm{~mA}$ and $100 \mathrm{~mA}$. 
Table 1 CIE color coordinates of DCTP LEDs.

\begin{tabular}{lcc}
\hline Current $(\mathrm{mA})$ & $x$ & $y$ \\
\hline 10 & 0.362 & 0.391 \\
30 & 0.361 & 0.390 \\
50 & 0.350 & 0.385 \\
100 & 0.343 & 0.390 \\
\hline
\end{tabular}

DCTP structures have been intentionally designed to achieve white color emission from a single $3 \mathrm{D}$ structure that has a lower height. A DCTP structure has $\{10 \overline{1} 1\},\{11 \overline{2} 2\},\{11 \overline{2} 0\}$, and (0001) facets that lead to high CRI white emission ( $\mathrm{Ra} \sim 75 \%$ ). A CL experiment was conducted to determine the spatially resolved optical characterization. We confirm that each facet emits a different wavelength. HAADF-STEM images were taken to check the origin of the emission wavelength. We also conducted $\mu$-PL and $\mu$-EL measurements to assess the differences and found that the hole injection efficiency strongly depends on the thickness of the $p$-GaN layer for the 3D structure. This method could be adopted for checking the local hole injection for not only 3D structure LEDs but also 2D film LEDs. We successfully demonstrated a phosphor-free white LED, and the CIE color coordinates are relatively stable with varying current.

\section{ACKNOWLEDGEMENTS}

This work was supported by the National Research Foundation (NRF2013R1A2A1A01016914, NRF-2013R1A1A2011750) of the Ministry of Education, the Industrial Strategic Technology Development Program (10041878) of the Ministry of Knowledge Economy, the Climate Change Research Hub of KAIST (Grant No.N01150041), and the GRC project of KAIST Institute for the NanoCentury. The authors wish to thank Soo Kun Jeon and his colleagues (Semicon Light Co., Ltd., Republic of Korea) for their help with the device fabrication process.

1 Nakamura S, Mukai T, Senoh M. Candela-class high-brightness InGaN/AIGaN doubleheterostructure blue-light-emitting diodes. Appl Phys Lett 1994; 64: 1687-1689.

2 Ponce FA, Bour DP. Nitride-based semiconductors for blue and green light-emitting devices. Nature 1997; 386: 351-359.

3 Schubert EF, Kim JK. Solid-state light sources getting smart. Science 2005; 308 : 1274-1278.

4 Nguyen HPT, Zhang SF, Connie AT, Kibria MG, Wang Q et al. Breaking the carrier injection bottleneck of phosphor-free nanowire white light-emitting diodes. Nano Lett 2013; 13: 5437-5442.

5 Li YL, Gessmann T, Schubert EF, Sheu JK. Carrier dynamics in nitride-based lightemitting $\mathrm{p}-\mathrm{n}$ junction diodes with two active regions emitting at different wavelengths. J Appl Phys 2003; 94: 2167-2172.
6 Park IK, Kim JY, Kwon MK, Cho CY, Lim JH et al. Phosphor-free white light-emitting diode with laterally distributed multiple quantum wells. App/ Phys Lett 2008; 92: 091110.

7 Soh CB, Liu W, Teng JH, Chow SY, Ang SS et al. Cool white III-nitride light emitting diodes based on phosphor-free indium-rich InGaN nanostructures. Appl Phys Lett 2008; 92: 261909.

8 Cho CY, Park IK, Kwon MK, Kim JY, Park SJ et al. InGaN/GaN multiple quantum wells grown on microfacets for white-light generation. Appl Phys Lett 2008; 93: 241109.

9 Wunderer T, Wang J, Lipski F, Schwaiger S, Chuvilin A et al. Semipolar GalnN/GaN light-emitting diodes grown on honeycomb patterned substrates. Phys Stat Solidi $C$ 2010; 7: 2140-2143.

$10 \mathrm{Kim}$ JH, Ko YH, Cho JH, Gong SH, Ko SM et al. Toward highly radiative white light emitting nanostructures: a new approach to dislocation-eliminated GaN/InGaN coreshell nanostructures with a negligible polarization field. Nanoscale 2014; 6: 1421314220.

11 Ko YH, Song J, Leung B, Han J, Cho YH. Multi-color broadband visible light source via GaN hexagonal annular structure. Sci Rep 2014; 4: 5514.

12 Wu K, Wei TB, Zheng HY, Lan D, Wei XC et al. Fabrication and optical characteristics of phosphor-free InGaN nanopyramid white light emitting diodes by nanospherical-lens photolithography. J App/ Phys 2014; 115: 123101.

13 Zhu D, Wallis DJ, Humphreys CJ. Prospects of III-nitride optoelectronics grown on Si. Rep Prog Phys 2013; 76: 106501.

14 Chichibu SF, Uedono A, Onuma T, Haskell BA, Chakraborty A et al. Origin of defectinsensitive emission probability in In-containing (AI,In,Ga)N alloy semiconductors. Nat Mater 2006; 5: 810-816.

15 Schwarz UT, Kneissl M. Nitride emitters go nonpolar. Phys Stat Solidi RRL 2007; 1: A44-A46.

16 Tsuchiya T, Shimizu J, Shirai M, Aoki M. InGaAlAs selective-area growth on an InP substrate by metalorganic vapor-phase epitaxy. J Cryst Growth 2005; 276: 439-445.

17 Feng W, Kuryatkov VV, Chandolu A, Song DY, Pandikunta M et al. Green light emission from InGaN multiple quantum wells grown on GaN pyramidal stripes using selective area epitaxy. J Appl Phys 2008; 104: 103530.

18 Fujii T, Gao Y, Sharma R, Hu EL, DenBaars SP et al. Increase in the extraction efficiency of GaN-based light-emitting diodes via surface roughening. Appl Phys Lett 2004; 84: 855-857.

19 Tchoe Y, Jo J, Kim M, Heo J, Yoo G et al. Variable-color light-emitting diodes using GaN microdonut arrays. Adv Mater 2014; 26: 3019-3023.

20 Hong YJ, Lee CH, Yoon A, Kim M, Seong HK et al. Visible-color-tunable light-emitting diodes. Adv Mater 2011; 23: 3284-3288.

21 Scholz F. Semipolar GaN grown on foreign substrates: a review. Semicond Sci Technol 2012; 27: 024002.

22 Amari H, Ross IM, Wang T, Walther $T$. Characterization of InGaN/GaN epitaxial layers by aberration corrected TEM/STEM. Phys Stat Solidi C 2012; 9: 546-549.

23 Leung B, Sun Q, Yerino CD, Han J, Coltrin ME. Using the kinetic Wulff plot to design and control nonpolar and semipolar GaN heteroepitaxy. Semicond Sci Technol 2012; 27: 024005.

24 Ko SM, Gong SH, Cho YH. Nonlinear photonic diode behavior in energy-graded coreshell quantum well semiconductor rod. Nano Lett 2014; 14: 4937-4942.

\section{(c) (i) $(-)$ This work is licensed under a Creative Commons Attribution-}

cc) NonCommercial-NoDerivs 4.0 Unported License. The images or other third party material in this article are included in the article's Creative Commons license, unless indicated otherwise in the credit line; if the material is not included under the Creative Commons license, users will need to obtain permission from the license holder to reproduce the material. To view a copy of this license, visit http://creativecommons.org/licenses/ by-nc-nd/4.0/ 